\title{
KINEMATIC SYNTHESIS OF LINKAGE MECHANISMS USING BURMESTER POINTS AT THE GIVEN DWELL DURATION OF THE OUTPUT LINK
}

\author{
Viacheslav Oleksandrovych Kharzhevskyi ${ }^{1}$ \\ 1 Department of Mechanical Engineering, Khmelnytskyi National University, Instytutska str. 11, Khmelnytskyi \\ 29016, Ukraine, e-mail: vk@solidworks.net.ua
}

Received: 2016.11.31

Accepted: 2017.02.13

Published: 2017.06.01

\begin{abstract}
The paper is dedicated to the kinematic synthesis of dwell linkage mechanisms on the basis of the four-bar linkage. As known, to obtain the dwell of the output link, linkage mechanisms can be used and they have a number of advantages in comparison with other types of mechanisms. Using the kinematic geometry methods, special points of the mechanism's coupler plane can be found. One of these points is Burmester point and it enables to synthesize four-bar linkages which coupler point traces the curve, a part of which is approximately close to the arc of the circle. By joining the additional structural group, dwell linkage mechanism can be designed. But the problem is to find such parameters of the mechanism which allow designing dwell linkages with a given duration of the dwell. Using the presented numerical and analytical method, this problem was solved; the samples of reference diagrams for the synthesis are also presented in the article. The method enables to carry out kinematic synthesis of dwell mechanisms, taking into account a number of other parameters, such as the length of the links, maximum displacement of the output link, dwell exactitude etc.
\end{abstract}

Keywords: kinematic synthesis, dwell linkages, path generating, kinematic geometry, Burmester points, coupler curves.

\section{INTRODUCTION}

The mechanisms with a periodic dwell of the output link during continuous rotation of the input link are widely used in different fields of machinery. For this purpose, the various types of mechanisms can be used, for example, cam mechanisms, Geneva-type mechanisms etc. But with the defined sizes of links, this problem can be solved with linkage mechanisms which are composed of rigid bodies, have only lower kinematic pairs and geometric closure of the links. It enables to significantly increase the working velocities of machines, their durability and reliability, and also increase the load-carrying capacity, which is an important task in modern machine building.

Despite a number of advantages, the practical usage of linkage mechanisms is complicated due to their synthesis that is one of the most complex problems in the theory of mechanisms and machines.

As known, for designing the dwell mechanisms, path generating linkages can be used. In particular, basic circular and straight-line mechanisms can be used. For now, many methods of such mechanisms' synthesis are developed, which are within two main directions: the first one is the usage of algebraic Chebyshev's methods that use the conditions of the Chebyshev's best approximation of the functions and the second one is the usage of the kinematic geometry methods which are grounded by Burmester [2]. Further development of the Chebyshev's methods was carried out, in particular, by Blokh, Kinytskyi [4], Sarkissyan [10], Gassmann [3]. The kinematic geometry methods were further developed by Müller [9], Beyer, Lichtenheldt, Cherkudinov [1], Mur- 
ray [8], Yin, Han [11]. The modern state of the theory of synthesis of linkages is presented in the works of McCarthy [7], Wang [12].

According to the curvature theory, the main principle of the synthesis by kinematic geometry methods is to find such special points in the coupler plane of the mechanism which are the multiple knots of interpolation, and which satisfy the criteria of having high order tangency of coupler curve to its tangent circle in this point. One of such special points of the coupler plane that can be used to synthesize circular path generating linkages with good approximation to an arc of the circle is Burmester point, which is defined for the five infinitesimally close positions of the coupler.

But despite the large amount of methods, the problem is to predict precisely the dwell duration of the output link of the mechanism that is synthesized using Burmester point and to synthesize such mechanism using a given value of the dwell.

The goal of the article is to define the areas of parameters' existence of such mechanisms and to give the designers a convenient numerical and analytical method which allows defining the

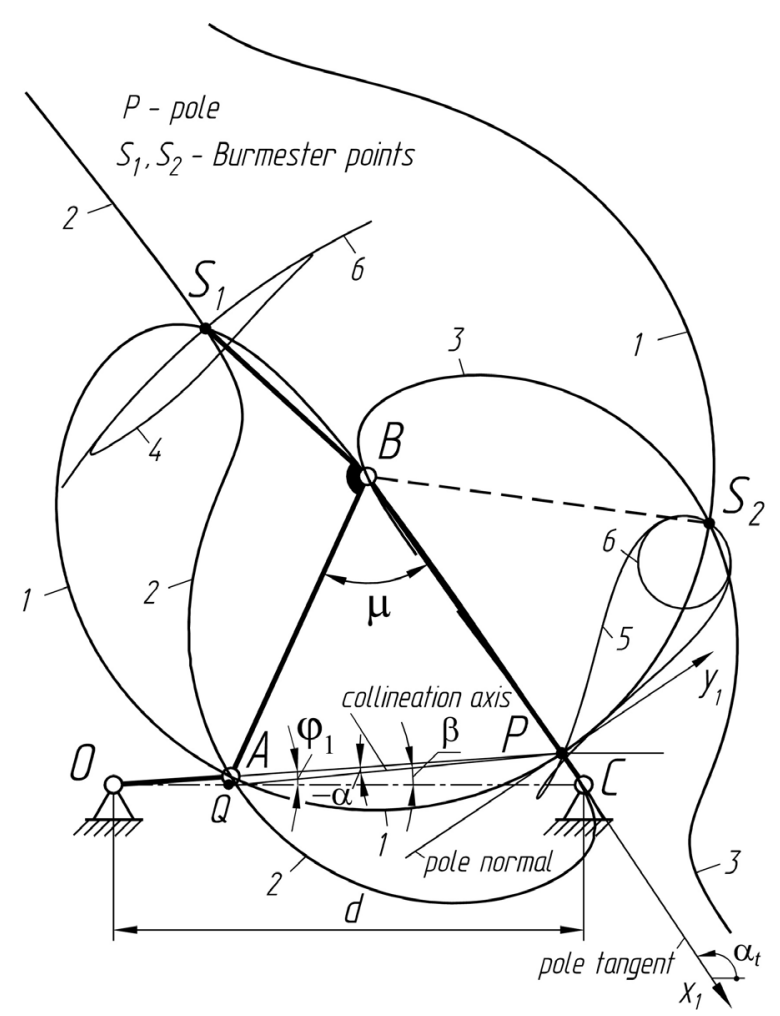

Fig. 1. Four-bar linkage with Burmester points of its coupler plane: 1 - circle point curve; 2,3- locus of the points with $4^{\text {th }}$ order of tangency; 4,5- coupler curves of Burmester points; 6 - approximation arcs necessary geometrical parameters taking into account a number of additional characteristics of a designed mechanism.

\section{KINEMATIC GEOMETRY METHODS}

Let us consider a circular path generating four-bar linkage (fig. 1). The mechanism contains the crank $r=l_{O A}$, the coupler $b=l_{A B}$ and the rocker $c=l_{B C}$. All the lengths are relative, the distance $\mathrm{d}=1_{\mathrm{OC}}=1$. During the continuous rotation of the input link $O A$, the point $S_{1}$ (or $S_{2}$ ) of the coupler $A B$ traces a path, whose part is approximate to an arc.

All the constructions in kinematic geometry apply only to a particular position of the mechanism, which is defined by the angle $\varphi$ of crank $O A$ rotation. As shown in Fig. 1, two Burmester points can be found in the particular position of the coupler plane: those points are designate as $S_{1}$ and $S$. Circular path generating mechanism can be designed if we use one of these points as coupler point of the mechanism.

As the coupler point move on its path, the curvature of that path will change, and it will pass through maximum and minimum values at which its derivative will be zero. These points are called stationary or circle points. Their locus for a particular position of the mechanism is called cubic of stationary curvature or circle point curve 1 (Fig. 1). Near a stationary point the rate of change of curvature will be small and, thus, the path will have good approximation to the circle. All the circle points have the $3^{\text {rd }}$ order of tangency with their tangent circles (the curvature and its derivative equals to zero in this point). If simplified $[1,5]$, the equation of that curve in $\mathrm{x}_{1} \mathrm{Py}_{1}$ coordinate system can be written as follows:

$$
\left(x^{2}+y^{2}\right)\left[\left(x_{0}^{\prime \prime \prime}+3 y_{0}^{\prime \prime}\right) x+y_{0}^{\prime \prime \prime} y\right]-3 y_{0}^{\prime \prime 2} x y=0
$$

where $y^{\prime \prime}{ }_{0}, x_{0}^{\prime \prime \prime}, y^{\prime \prime \prime}{ }_{0}$ - the acceleration and $3^{\text {rd }}$ order derivatives of the pole $P$ displacement; $x^{\prime \prime \prime}{ }_{0}=0$.

The particular case of stationary point is Burmester point which has the $4^{\text {th }}$ order of tangency to its tangent circle. In this case, the curvature and its two derivatives in this point equal zero. The locus of the points with the $4^{\text {th }}$ order of tangency lies on appropriate curve 2. Its equation in implicit form is $[1,5]$ :

$$
\begin{aligned}
& \left(x^{2}+y^{2}\right)\left(m_{3}+m_{4} x+m_{5} y\right)- \\
& -y_{0}^{\prime \prime} y\left(m_{1} x+m_{2} y+m_{3}\right)=0
\end{aligned}
$$


where coefficients $m_{1}, \ldots, m_{5}$ are:

$$
\begin{aligned}
& m_{1}=4 \omega y_{0}^{\prime \prime \prime} ; m_{2}=-4 x_{0}^{\prime \prime \prime}-6 y_{0}^{\prime \prime} ; m_{3}=3 y_{0}^{\prime \prime 2} \\
& m_{4}=x_{0}^{I V}+m_{1} ; m_{5}=y_{0}^{I V}+y_{0}^{\prime \prime}+m_{2}
\end{aligned}
$$

The parametric equation of (2) which enabled to build this curve was also obtained and given in [6].

Thus, Burmester points can be found as intersection points of the curve (1) and (2). The values of the $3^{\text {rd }}$ order derivatives $\mathrm{x}^{\prime \prime \prime}{ }_{0}, \mathrm{y}^{\prime \prime \prime}{ }_{0}$ of the pole $P$ displacement, which are in (3), can be found as follows:

$$
\begin{gathered}
x_{0}^{\prime \prime \prime}=3 y_{A} y_{0}^{\prime \prime 2} /\left(x_{A}^{2}+y_{A}^{2}\right)-y_{A} y_{0}^{\prime \prime \prime} / x_{A}-3 y_{0}^{\prime \prime} \\
y_{0}^{\prime \prime \prime}=\frac{3 x_{A} x_{B} y_{B} y_{0}^{\prime \prime 2}\left(y_{B}-y_{A}\left(x_{B}^{2}+y_{B}^{2}\right) /\left(x_{A}^{2}+y_{A}^{2}\right)\right)}{\left(x_{A} y_{B}-x_{B} y_{A}\right)\left(x_{B}^{2}+y_{B}^{2}\right)}
\end{gathered}
$$

The $4^{\text {th }}$ order derivatives $y^{I V}, y^{I V}{ }_{0}$ which are in (3):

$$
\begin{gathered}
x_{0}^{I V}=\frac{y_{A} y_{0}^{\prime \prime}\left[3 y_{0}^{\prime \prime}-y_{A}\left(4 x_{0}^{\prime \prime \prime}+6 y_{0}^{\prime \prime}\right)+4 x_{A} y_{0}^{\prime \prime \prime}\right]}{x_{A}\left(x_{A}^{2}+y_{A}^{2}\right)}- \\
-\left[3 y_{0}^{\prime \prime}-y_{A}\left(4 x_{0}^{\prime \prime \prime}+5 y_{0}^{\prime \prime}-y_{0}^{I V}\right)\right] / x_{A}-4 y_{0}^{\prime \prime \prime} \\
y_{0}^{I V}=-x_{A} n_{1} /\left(x_{A} y_{B}-x_{B} y_{A}\right)+x_{A} y_{B} y_{0}^{\prime \prime} \times \\
\times \frac{3 y_{0}^{\prime \prime}-y_{B}\left(4 x_{0}^{\prime \prime \prime}+6 y_{0}^{\prime \prime}\right)+4 x_{B} y_{0}^{\prime \prime \prime}}{\left(x_{A} y_{B}-x_{B} y_{A}\right)\left(x_{B}^{2}+y_{B}^{2}\right)}
\end{gathered}
$$

where $x_{A}, y_{A}, x_{B}, y_{B}$ - coordinates of the moving pivots $A$ and $B$ in the coordinate system $x P y$. Coefficient $n$ in equation (7) can be found as follows:

where:

$$
\begin{aligned}
& n_{1}=x_{B} y_{A} y_{0}^{\prime \prime}\left(n_{2}-y_{A} y_{0}^{\prime \prime}\right) / x_{A}\left(x_{A}^{2}+y_{A}^{2}\right)- \\
& -x_{B} n_{2} / x_{A}+4 y_{0}^{\prime \prime \prime} x_{B}+3 y_{0}^{\prime \prime 2}-y_{B}\left(4 x_{0}^{\prime \prime \prime}+5 y_{0}^{\prime \prime}\right)
\end{aligned}
$$

$$
n_{2}=3 y_{0}^{\prime \prime 2}+4 x_{A} y_{0}^{\prime \prime \prime}-y_{A}\left(4 x_{0}^{\prime \prime \prime} y_{A}+5 y_{0}^{\prime \prime}\right)
$$

As stated in the theoretical grounds of the kinematic geometry $[1,5]$, in particular position of the coupler plane of the four-bar linkage, two or four Burmester points can be found. As far as the moving pivots $A$ and $B$ are also considered as Burmester points, because those trajectories are ideal arcs (i.e. have not only $4^{\text {th }}$ but any higher order of tangency with a circle), so maximum two Burmester points can be used for designing circular path generating linkages.

\section{PARAMETRIC SYNTHESIS OF DWELL LINKAGES}

As known, the mechanisms with periodic dwell of the output link can be synthesized on the basis of path generating linkages. Such a mecha- nism is shown in Fig. 2: it consists of the basic four-bar linkage with joined additional structural group 4-5. The mechanism works in the way that during the continuous rotation of the crank 1 , the coupler point $D$ traces the curve which approximates to an arc in the part $D^{\prime} D^{\prime \prime}$. The length $R$ $=l_{D E}$ of the coupler 4 equals to the radius of the approximation arc, thus while point $D$ moves on the part $D^{\prime} D^{\prime \prime}$, the output link 5 has a dwell. Its duration $\alpha_{\Sigma}$ equals to the crank rotation angle $\varphi_{1}$ which corresponds to the dwell period.

Let us consider the values of $r, b, c$ and angle of crank rotation $\varphi$ as given, thus to design a dwell linkage mechanism we should first define the coordinates of the point $A$, in the coordinate system $x O y$ :

$$
X_{A}=r \cos \varphi_{1} ; \quad Y_{A}=r \sin \varphi_{1}
$$

The angles $\varphi_{2}$ and $\varphi_{3}$ which define positions of the coupler 2 and rocker 3 of the mechanism:

$$
\varphi_{2}=\psi+\kappa ; \varphi_{3}=\psi-\chi+\pi
$$

where $\Psi=\operatorname{arctg}\left[\mathrm{Y}_{\mathrm{A}} /\left(\mathrm{X}_{\mathrm{A}}-1\right)\right] ; \chi=\pi-\kappa-\mu$.

To define the required angles in (9), we should additionally define such parameters as [5]:

$$
\begin{aligned}
& \kappa=\arccos \left[\left(b^{2}+\Delta^{2}-c^{2}\right) / 2 b \Delta\right] \\
& \mu=\arccos \left[\left(b^{2}+c^{2}-\Delta^{2}\right) / 2 b c\right]
\end{aligned}
$$

where:

$$
\Delta=\sqrt{\left(1-X_{A}\right)^{2}+Y_{A}^{2}}
$$

The coordinates of the point $B$ of the mechanism:

$$
X_{B}=1+c \cos \varphi_{3} ; Y_{B}=c \sin \varphi_{3}
$$

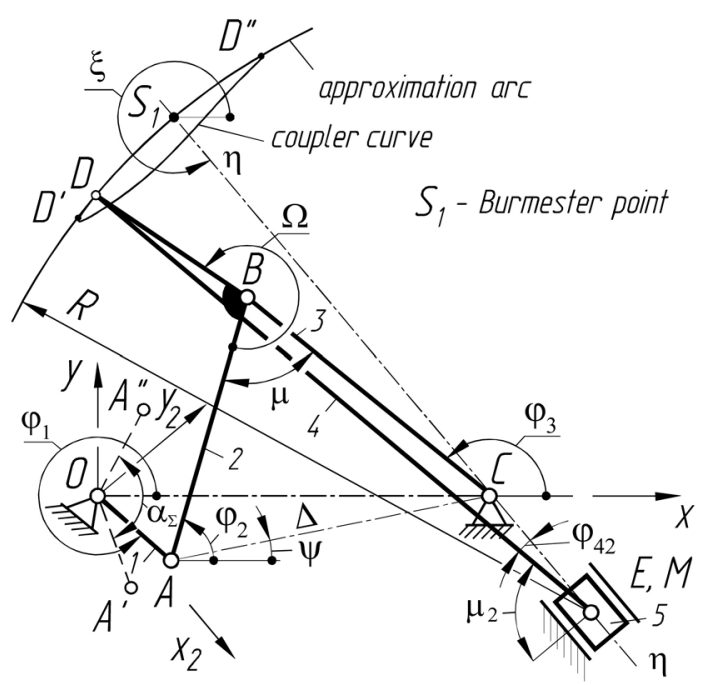

Fig. 2. Dwell linkage mechanism synthesized using Burmester point 
The coordinates of the poles $P$ and $Q$ [1] (Fig. 1):

$$
\begin{aligned}
& X_{P}=\operatorname{tg} \varphi_{3} /\left(\operatorname{tg} \varphi_{3}-\operatorname{tg} \varphi_{1}\right) ; Y_{P}=X_{P} \operatorname{tg} \varphi_{1} \\
& X_{Q}=\left(X_{A} Y_{B}-X_{B} Y_{A}\right) /\left(Y_{B}-Y_{A}\right) ; Y_{Q}=0
\end{aligned}
$$

To define the parameters of the curves (1) and (2), we should find the pole $P$ acceleration $[1,5]$ :

$$
y_{0}^{\prime \prime}=-\left|l_{O P}\left(l_{O P}-r\right) / r \sin \left(\varphi_{3}-\beta\right)\right|
$$

where $l_{O P}=X_{p} / \cos \varphi_{1}$, the angle of collineation axis $\beta=\arctan \left[Y_{p} /\left(Y_{p}-X_{Q}\right)\right]$.

If the basic coordinate system $x O y$ is rotated to $x_{1} P y_{1}$ then $\mathrm{x}_{0}{ }_{0}=0$ and it simplifies the formulae. Therefore, we should calculate the coordinates of the pivots $A$ and $B$ in rotated coordinate system $x_{1} P y_{1}[1]$ :

$$
\begin{aligned}
& x=\left(X_{P}-X\right) \cos \alpha_{t}+\left(Y_{P}-Y\right) \sin \alpha_{t} \\
& y=\left(Y_{P}-Y\right) \cos \alpha_{t}+\left(X-X_{P}\right) \sin \alpha_{t}
\end{aligned}
$$

where $X, Y$ - coordinates of the pivots $A$ or $B$ in $x O y ; x$ and $y$ - required coordinates of the pivots in $x_{1} P y_{1}$ the angle of pole tangent $\alpha_{t}=\varphi_{1}+\varphi_{3}-\beta$.

The system of equations (1) and (2) which defines Burmester points, have 4 roots. Two of which correspond to the pivots $A$ and $B$ of the mechanism:

$$
x_{1}=\tan \left(\varphi_{1}-\varphi_{3}-\alpha\right) ; x_{2}=-\tan \alpha ; \alpha=\varphi_{1}-\beta
$$

Another two roots define Burmester points:

$$
x_{3,4}=\left(-p \pm \sqrt{p^{2}-4 q}\right) / 2
$$

where coefficients $p$ and $q$ :

$$
\begin{gathered}
p=3 m_{2} y_{0}^{\prime \prime 2} / m_{3} y_{0}^{\prime \prime \prime}+\left(3 y_{0}^{\prime \prime}+2 x_{0}^{\prime \prime \prime}\right) / y_{0}^{\prime \prime \prime}+x_{1}+x_{2} \\
q=p\left(x_{1}+x_{2}\right)-x_{1} x_{2}+\left(3 y_{0}^{\prime \prime} x_{0}^{\prime \prime \prime}+x_{0}^{\prime \prime 2}+y_{0}^{\prime \prime 2}\right) / y_{0}^{\prime \prime 2}- \\
-3 y_{0}^{\prime \prime 2}\left[3 y_{0}^{\prime \prime}\left(m_{5}-m_{2}\right)-m_{2} x_{0}^{\prime \prime \prime}-m_{1} y_{0}^{\prime \prime \prime}\right] / m_{3} y_{0}^{\prime \prime 2}
\end{gathered}
$$

Finally, the coordinates of the Burmester points:

$$
x_{S_{1,2}}=\frac{m_{3}\left(x_{0}^{\prime \prime \prime}+y_{0}^{\prime \prime \prime} x\right)}{n_{4}-x n_{3}-m_{2} y_{0}^{\prime \prime} x^{2}} ; y_{S_{1}}=x_{S_{1}} x
$$

where $\mathrm{x}=\mathrm{x}_{3}$ for the $\mathrm{S}_{1}$ and $\mathrm{x}=\mathrm{x}_{4}$ for the $\mathrm{S}_{2}$;

$$
\begin{gathered}
n_{3}=3 y_{0}^{\prime \prime}\left(m_{5}-m_{2}\right)-m_{2} x_{0}^{\prime \prime \prime}-m_{1} y_{0}^{\prime \prime \prime} \\
n_{4}=3 y_{0}^{\prime \prime}\left(m_{4}-m_{1}\right)-m_{1} x_{0}^{\prime \prime \prime}
\end{gathered}
$$

To find the position of Burmester points in $\mathrm{xOy}$, let's rotate back the coordinate system:

$$
\left.\begin{array}{l}
X_{S_{1,2}}=X_{P}-x_{S_{1,2}} \cos \alpha_{t}+y_{S_{1,2}} \sin \alpha_{t} ; \\
Y_{S_{1,2}}=Y_{P}-y_{S_{1,2}} \cos \alpha_{t}-x_{S_{1,2}} \sin \alpha_{t} .
\end{array}\right\}
$$

Having found the coordinates of each Burmester point, we can define the parameters of the coupler 2 :

$$
\begin{aligned}
& k=l_{B D}=\sqrt{\left(X_{S_{1,2}}-X_{B}\right)^{2}+\left(Y_{S_{1,2}}-Y_{B}\right)^{2}} \\
& \Omega=\pi-\varphi_{2}+\arctan \left(Y_{S_{1,2}}-Y_{B} / X_{S_{1,2}}-X_{B}\right)
\end{aligned}
$$

The coordinates of the centers $M$ of approximation arcs and their radii:

$$
\begin{aligned}
& x_{M_{1,2}}=\frac{3 x y_{0}^{\prime \prime 2}}{\left(x^{2}+1\right)\left(x_{0}^{\prime \prime}+y_{0}^{\prime \prime} x\right)} ; y_{M_{1,2}}=x_{M_{1,2}}{ }^{2} \\
& R_{1,2}=\sqrt{\left(X_{M_{1,2}}-X_{S_{1,2}}\right)^{2}+\left(Y_{M_{1,2}}-Y_{S_{1,2}}\right)^{2}}
\end{aligned}
$$
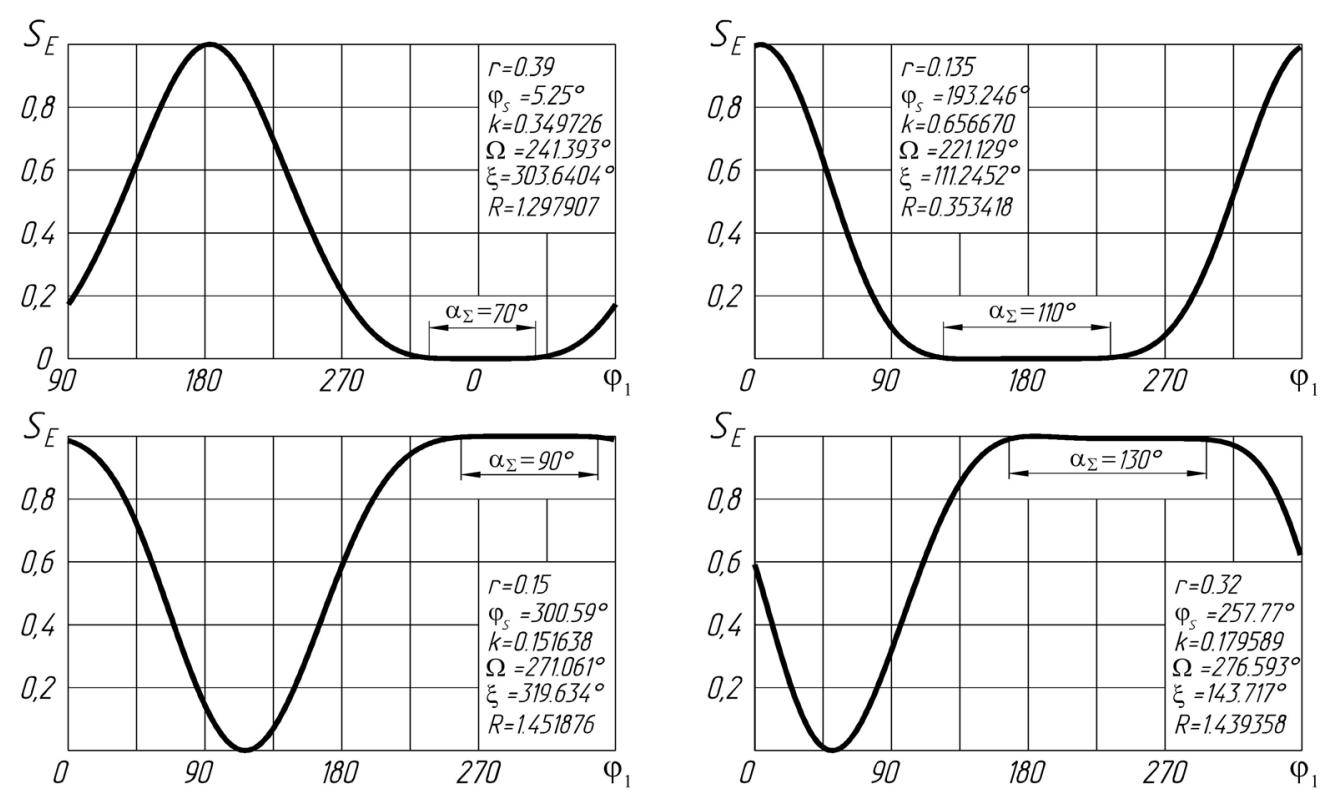

Fig. 3. The samples of displacement diagrams of the output link: dwell duration was defined using the numerical method [5] by means of the unitless coefficient of maximum velocity 

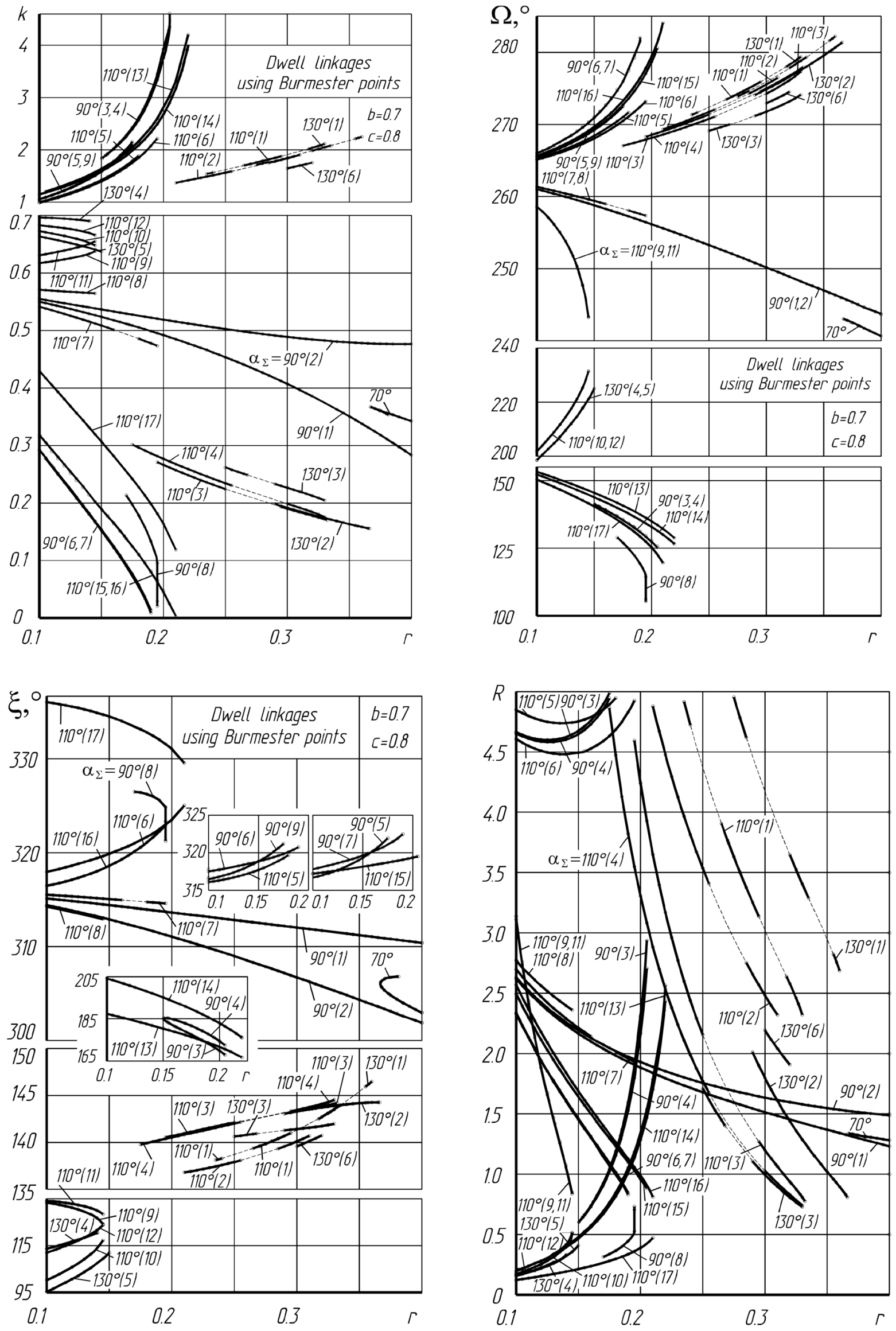

Fig. 4. The reference diagrams for the geometric parameters' determination at the given duration of the dwell 
where $\mathrm{x}=\mathrm{x}_{3}$ for the $\mathrm{M}_{1}$ and $\mathrm{x}=\mathrm{x}_{4}$ for the $\mathrm{M}_{2}, \mathrm{X}_{\mathrm{M}}$, $\mathrm{Y}_{\mathrm{M}}$ - coordinates of the point $M$ in $x O y$, which are calculated in the same way as (18).

The angle $\xi$ of the guiding line $\eta-\eta$ of the slider 5:

$$
\xi_{1,2}=\arctan \left(Y_{S_{1,2}}-Y_{M_{1,2}} / X_{S_{1,2}}-X_{M_{1,2}}\right)
$$

To define the displacement of the output link 5 , we should firstly find the position of the point $D$ in $x O y$ :

$$
X_{D}=X_{B}-k \cos \left(\varphi_{2}+\Omega\right) ; Y_{D}=Y_{B}-k \sin \left(\varphi_{2}+\Omega\right)
$$

For the calculation of the parameters of the joined structural group 4-5, it is convenient to rotate the coordinate system $x O y$ on the angle $\xi$ and find the parameters in the coordinate system $x_{2} \mathrm{Oy}_{2}$ :

$$
\left.\begin{array}{l}
x_{D 2}=x_{D} \cos \xi+y_{D} \sin \xi ; \\
y_{D 2}=y_{D} \cos \xi-x_{D} \sin \xi .
\end{array}\right\}
$$

Finally, the coordinate of the output link 5 which defines its displacement $S_{E}$ :

$$
x_{E_{2}}=x_{D_{2}}+R \cos \varphi_{42}
$$

where the angle that defines the position of the coupler 4: $\varphi_{42}=\arcsin \left[\left(\mathrm{y}_{\mathrm{M} 2}-\mathrm{y}_{\mathrm{D} 2}\right) / \mathrm{R}\right], \mathrm{y}_{\mathrm{M} 2}-$ ordi- nate of the point $M$, which is the center of the approximation arc (20), but in rotated coordinate system $x O y$, transformed in the same way as in (22).

$U^{2} \operatorname{sing}$ the formulae (3)-(23) we can define all the necessary geometric parameters of the linkage mechanism with a dwell of the output link. Each combination of input parameters $\left(r, b, c, \varphi_{1}\right)$ will define different mechanisms with unknown dwell period. So, first, we should solve a problem of determining the actual dwell period which corresponds to the initial synthesis parameters. For this purpose, a numerical method was used. The method is based on the usage of unitless coefficient of maximum velocity as proposed in the work [5], where it was used for the synthesis of straight-line mechanisms. It was established that the mentioned method can also be used for mechanisms considered in this article. As shown in the Fig. 3, the dwell time of the output link is correctly defined for different durations of the dwell.

Another problem is to solve a reverse task define such parameters of the mechanism that allow obtaining the given value of the dwell. This problem was also solved numerically and it allows making up the reference diagrams taking
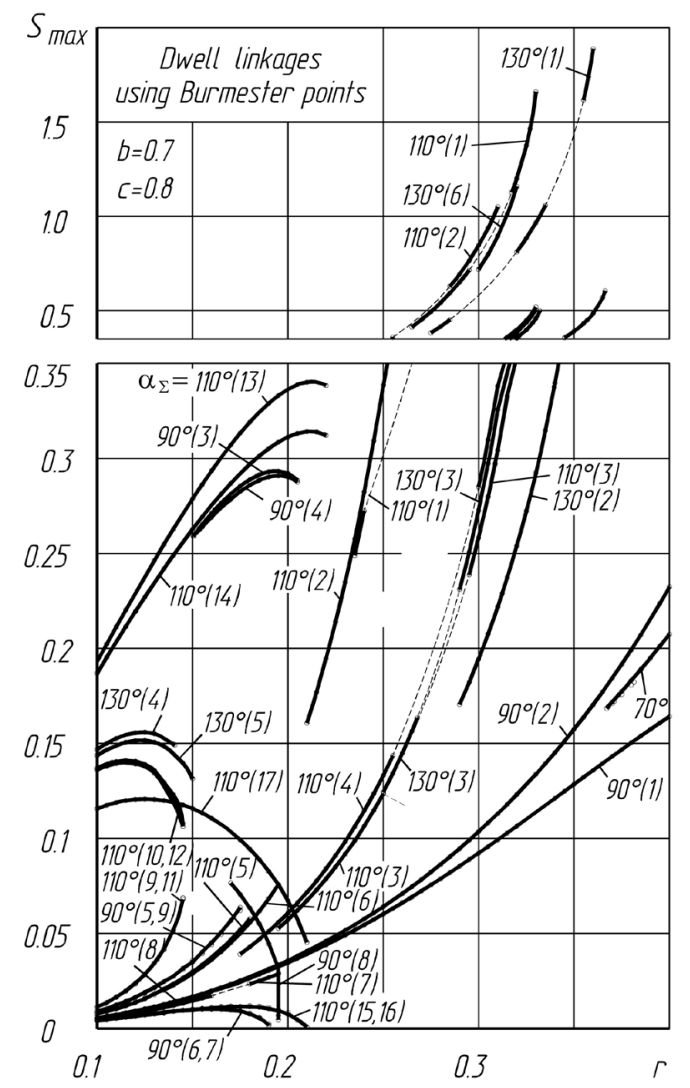

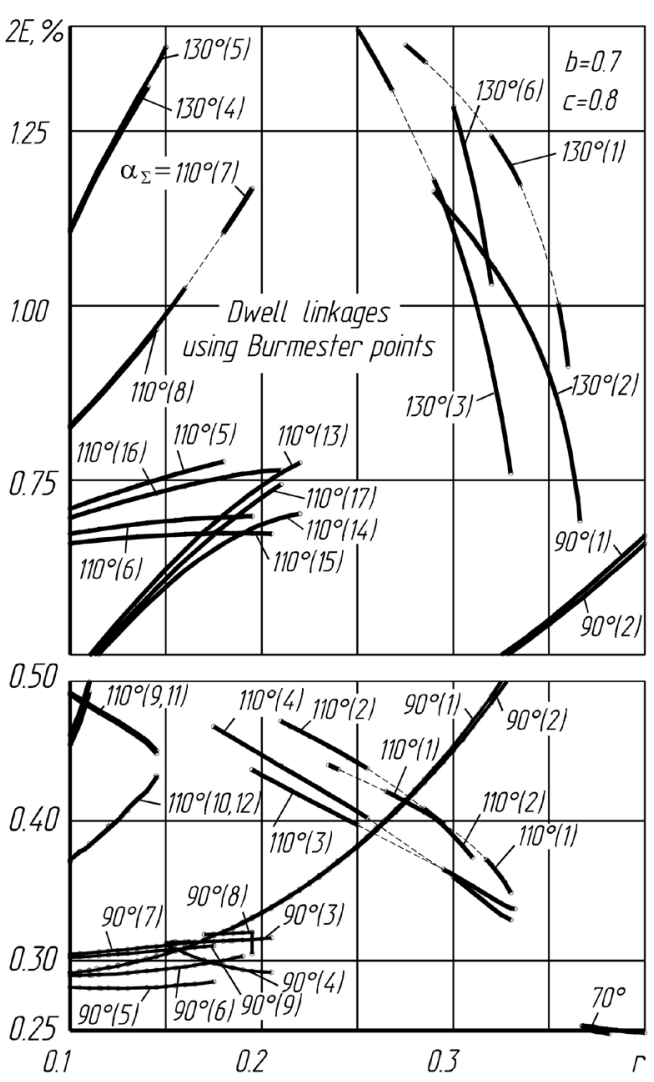

Fig. 5. The reference diagrams for the determination of the maximum displacement $S_{\max }$ of the output link and the exactitude $2 E$ of the dwell (in percents of maximum displacement) 
into account the different parameters of designed mechanisms. The samples of such reference diagrams are shown in the Figure 4 and Figure 5.

On the basis of the presented method of the dwell mechanisms' synthesis, the algorithms and correspondent software were developed. Fig. 4,5 shows only those diagrams which allow to define parameters of the mechanisms with dwell duration equals to $\alpha_{\Sigma}=70^{\circ}, 90^{\circ}, 110^{\circ}, 130^{\circ}$ and with such lengths of the basic four-bar linkage as: $\mathrm{b}=0.7 ; \mathrm{c}=0.8 ; \mathrm{r}=0.1 \ldots 0.4$. As it was defined, almost for each dwell duration, the area of existence consists of several series of graphs. Every graph in the diagrams has its number in brackets which helps to find a correspondent graph in other diagrams, and thus define all the necessary parameters of the desired mechanism.

As shown above, for a particular position of the coupler plane, two Burmester points can be found, or those points do not exist (if not count the moving pivots $A$ and $B$ of the mechanism). Besides, not all Burmester points can be used for designing the dwell mechanisms, because it is not always possible to join additional structural group 4-5 due to the inappropriate radius $R$ of approximation. Thus, only those Burmester points that can be used to make dwell linkages were used to establish the reference diagrams (Fig. 4 and Fig. 5).

Developed software allows building reference diagrams at any other initial parameters of synthesis besides the ones shown on Fig. 4 and Fig. 5. These diagrams actually show the areas of parameters' existence and it can help designer to preliminarily define the parameters of the linkage at the first stage of projection. Then, by means of developed software, the geometric parameters can be defined more precisely, as shown, for example, in the Fig. 3.

All the parameters of the synthesized mechanisms are recorded into the database and optimization procedures of kinematic synthesis using different criteria are also performed. The synthesis method that is proposed in the article can be used not only for the synthesis of the dwell mechanisms, but also for the four-bar circular path generating linkage mechanisms. Their synthesis is an independent task which is also important in different fields of machine designing.

\section{CONCLUSIONS}

The problem of synthesis of dwell linkage mechanisms on the basis of path generating mech- anisms is so important in order to design more reliable mechanisms with higher working velocities and load-carrying capacity. The proposed method of their synthesis can help designer find optimal variant of mechanism at a given duration of the dwell of the output link taking into account a number of other parameters. Research is planned to be extended in the direction of optimal synthesis taking into account the main kinematic and dynamic characteristics of the linkage mechanisms.

\section{REFERENCES}

1. Artobolevsky I.I., Levitsky N.I., Cherkudinov S.A. Synthesis of planar mechanisms - Moscow: Fizmatgiz, 1959 [in Russian].

2. Burmester L. Lehrbuch der Kinematik. Felix Verlag, Leipzig, Germany 1888.

3. Gassmann V. Synthese von Geradführungen mit ebenen Viergelenkgetrieben, Hamburg, Universität der Bundeswehr Diss., 2000.

4. Kinytskyi Ya. T. Chebyshev's linkages with dwell of the output link. Kyiv: Vyshcha shkola, 1990 [in Russian].

5. Kharzhevskyi V.O. Synthesis of the linkage mechanisms with dwell of the output links using kinematic geometry methods: monography. Khmelnytskyi RVC KhNU, 2015 [in Ukrainian].

6. Kharzhevskyi V.O. The method of determining of coupler points' position for the designing of the linkage straight-line mechanisms //Herald of Gomel State Technical University named after P.O.Sukhoi. 2015. 4(63), 7-13 [in Russian].

7. McCarthy, J., Soh G. Geometric Design of Linkages, 2nd edition. Springer-Verlag, New York, 2011.

8. Murray A., McCarthy J. Determining Burmester points from the analysis of a planar platform // Trans. ASME J. Mech. Des. - 1995, Vol. 117, 303-307.

9. Müller R. Konstruktion der Burmesterschen Punkte für ein ebenes Gelenksviereck, Zeitschrift Mathematik und Physik, 1892.

10. Sarkissyan Y.L. Approximations in Synthesis of Mechanisms /State Engineering University of Armenia Proceedings, series "Mechanics, Machine Science, Machine-building", Issue 15(2), 2012, 9-21.

11. Yin L., Han J., Huang J., Yang T. "A General Method for Synthesizing Straight-Line Linkage with Ball and Burmester Points" //Applied Mechanics and Materials, Vols 215-216, 2012, 138-141.

12. Wang D., Wang W. Kinematic Differential Geometry and Saddle Synthesis of Linkages John Wiley \& Sons Singapore Pte. Ltd., 2015. 